\title{
Modeling of Microstructural Stresses of Composite Materials during Phase Transformations
}

\author{
E.M. Romanovskaia ${ }^{1, \text { a) }}$, E.A. Mityushov ${ }^{1, b)}$, S.A.Berestova ${ }^{1, c)}$, \\ N.D. Romanovskaia ${ }^{1, d)}$ \\ ${ }^{1}$ Ural Federal University, Mira 19, Yekaterinburg, Russia, 620002 \\ a)Corresponding author: E.M.Romanovskaia@urfu.ru \\ b)mityushov-e@mail.ru \\ ${ }^{c)}$ s.a.berestova@urfu.ru \\ d)NDRomanovskaya@yandex.ru
}

\begin{abstract}
Using the method of self-consistent field, we consider the problem of competing influence of structural components on the properties of the matrix arising in the transformation phase. To this end, the problem of determining the average stresses in the elements of the microstructure through the elastic characteristics and the concentrations of the matrix and phase components, as well as free deformations of the phases undergoing volume transformations, is previously solved. As a model, we consider a continuous matrix with isotropic elastic characteristics, into which spherical inclusions are introduced. The introduced inclusions are strongly intermixed and distributed equally. The matrix was assumed to be free from external loads, so the macroscopic stresses are zero. Under certain external influences, inclusions begin to undergo structural transformations with changes in the size and specific volume of the phase components, some of them begin to expand and others contract, which causes stresses in the composite structure that are balanced on a macroscopic scale. Following the method of the generalized self-consistent field, the average stresses over the volume occupied by the i-th dispersed phase are determined from the Eshelby solution on the deformation of a single particle. The collective influence of other dispersed particles is taken into account by the fact that the deformation in the matrix is different from zero and is assumed to be equal to the average deformation in the volume occupied by the matrix phase in the composite material. As a result of the transformations using the Eshelby tensor and the generalized Hooke's law, which is satisfied by the average stresses and strains in the matrix, a closed system of equations is obtained for determining the average stresses and deformations in the phase components, that are expressed in terms of the elastic characteristics and concentrations of the matrix and phase components as well as deformation of phases undergoing volume transformations.
\end{abstract}

\section{INTRODUCTION}

At present, structurally heterogeneous materials are increasingly used in structures subject to various force and temperature influences. Under the effect of these influences, internal stresses or microstresses may appear in the material, which are superimposed on the macroscopic stresses caused by the external load. In some cases, the combination of two stressed states can be dangerous. In this connection, it becomes necessary to study the behavior of a composite material and to determine the characteristics of stress fields and deformations in a microstructure under various phase transformations [1,2].

A method for determining structural stresses arising during phase transformations in a multiphase composite material, accompanied by a change in the volume of individual particles is proposed. The solution is based on the method of the generalized self-consistent field. 


\section{STATEMENT OF THE PROBLEM AND THE METHOD OF SOLVING IT}

As a model, we consider a continuous matrix with isotropic elastic characteristics, into which spherical inclusions with isotropic elastic properties are introduced. The introduced inclusions are strongly intermixed and distributed equally. The matrix is free from external loads, so the macroscopic stresses are zero. Under certain external influences, inclusions begin to undergo structural transformations with a change in the size and specific volume of the phase components, some of which begin to expand and others contract, causing stresses in the structure of the composite, balanced on a macroscopic scale

$$
\sum_{\mathrm{i}=1}^{\mathrm{n}} \mathrm{v}_{i} \boldsymbol{\sigma}^{(i)}+\mathrm{v}_{m} \boldsymbol{\sigma}^{m}=0
$$

where $\mathrm{v}_{i}$ and $\mathrm{v}_{m}$ are the volumetric concentrations of phase components and matrices, respectively; $\boldsymbol{\sigma}^{(i)}$ - tensors of average microstress in the $\mathrm{i}$-th phase component; $\boldsymbol{\sigma}^{m}$ - tensor of average microstresses in the matrix. Let us determine the stresses arising during phase transformations in a composite material.

The solution of this problem can be obtained from the solution of the problem of Eshelby [3] on the constrained deformation of an elastic inclusion placed in an isotropic matrix.

In the first stage, a spherical inclusion is distinguished from the homogeneous matrix-a region in a state free from bonds that undergoes phase transformations. As a result of the structural transformation, the size of the phase component changes, it undergoes free deformation - and returns to its original position (b-isotropic tensor of the 2nd rank). Let us now assume that the element under consideration is bond with neighboring elements acting on it prior to the structural transformation, then after structural transformation due to a geometric inconsistency between the dimensions of the inclusion and the cavity, a strained deformation arises in it, determined by the change in the region occupied by the inclusion and caused by this deformation some stress state.

The tensors of constrained deformation and free deformation are interconnected through the Eshelby tensor [4].

The resulting deformation of the particle is found as the difference between the strained and free: $\boldsymbol{\varepsilon}^{\boldsymbol{c}}-\mathbf{b}$. For the stresses arising in the particle, we write down the generalized Hooke's law

$$
\boldsymbol{\sigma}=\mathbf{C}:\left(\varepsilon^{c}-\mathbf{b}\right) \text {, }
$$

where $\mathbf{C}$ - is the elastic modulus tensor.

Since the material of the matrix and the elastic inclusion is isotropic, their properties are characterized by two independent constants: the volume modulus $K$ and the shear modulus $\mu$. Taking into account the expansions for the tensors of the elastic modulus and the Eshelby tensor we obtain the dependence for the stresses arising in a single spherical inclusion in the phase transformation

$$
\sigma_{i j}=\frac{2 b_{0} E}{9(1-v)} \delta_{i j}
$$

The collective influence of other dispersed particles is taken into account by the fact that the deformation in the matrix is different from zero and is assumed to be equal to the average deformation in the volume occupied by the matrix phase in the composite material.

As a result of the transformations using the Eshelby tensor, formulas (1), (2) and the generalized Hooke's law, which is satisfied by the average stresses and deformations in the matrix, a closed system of equations is obtained for determining the mean stresses and deformations in the phase components

$$
\left\{\begin{array}{c}
\sum_{i=1}^{n} \mathrm{v}_{i} \boldsymbol{\sigma}^{(i)}+\mathrm{v}_{m} \boldsymbol{\sigma}^{m}=0 \\
\boldsymbol{\sigma}^{(i)}=\mathbf{C}^{(i)}:\left(\mathbf{N}^{m}-\mathbf{I}\right): \mathbf{b}_{i}+\mathbf{C}^{(i)}:\left(\mathbf{C}^{m}\right)^{-1}: \boldsymbol{\sigma}^{m},
\end{array}\right.
$$

that are expressed through the elastic characteristics and concentrations of the matrix and phase components, as well as free deformations of the phases undergoing volumetric transformations.

\section{CALCULATION OF MICROSTRESSES IN PHASE TRANSFORMATIONS FOR A THREE-PHASE MATRIX SYSTEM.}

We use this solution to study the compensating effect of particles on the elastic properties of a heterogeneous material. Let $\boldsymbol{\sigma}^{(1)}, \boldsymbol{\sigma}^{(2)}, \boldsymbol{\sigma}^{m}$ be the microstresses arising in the phase components and in the matrix, respectively. In phase transformations, the field of average deformations in the matrix $\varepsilon_{m}$ is different from zero. In the first stage of the solution, we assume that the elastic characteristics of the matrix and the phase components coincide, i.e. $\mathbf{C}^{(1)}=$ $\mathbf{C}^{(2)}=\mathbf{C}^{(m)}=\mathbf{C}$.

Solving system (4), taking into account the formula (3) we obtain expressions describing the structural stresses arising in the phase components 


$$
\left\{\begin{array}{c}
\sigma_{i j}^{(1)}=-\frac{2 E}{9(1-v)} \delta_{i j}\left[b_{1}\left(1-\mathrm{v}_{1}\right)-b_{2} \mathrm{v}_{2}\right] \\
\sigma_{i j}^{(2)}=-\frac{2 E}{9(1-v)} \delta_{i j}\left[b_{2}\left(1-\mathrm{v}_{1}\right)-b_{1} \mathrm{v}_{2}\right] \\
\sigma_{i j}^{m}=-\frac{2 E}{9(1-v)} \delta_{i j}\left[b_{1} \mathrm{v}_{1}+b_{2} \mathrm{v}_{2}\right]
\end{array}\right.
$$

In the particular case of a two-phase system, assuming $b_{2}=0$ and $\mathrm{v}_{2}=0$ we obtain a result that coincides with the one found in the correlation approximation in the monograph [5]. Since no restrictions were imposed on the parameters of the statistical distribution of local characteristics in the derivation of equalities (5), this result is accurate in the framework of the model under consideration.

Now we consider the same problem for the case when the elastic properties of the matrix and the phase components differ from each other.

Passing from the tensor quantities to the scalar quantities, taking into account the expression of the Eshelby coefficients, we obtain for the stresses of the phase

$$
\left\{\begin{array}{l}
\sigma_{i j}^{(1)}=-\frac{2}{9}\left[\frac{2 v^{m}-1}{1-v^{m}} K^{(1)}\left(b_{1}-\frac{\left(\mathrm{v}_{1} K^{(1)} b_{1}+\mathrm{v}_{2} K^{(2)} b_{2}\right)}{\left(\mathrm{v}_{m} K^{m}+\mathrm{v}_{1} K^{(1)}+\mathrm{v}_{2} K^{(2)}\right)}\right)\right] \delta_{i j} \\
\sigma_{i j}^{(2)}=-\frac{2}{9}\left[\frac{2 v^{m}-1}{1-v^{m}} K^{(2)}\left(b_{2}-\frac{\left(\mathrm{v}_{1} K^{(1)} b_{1}+\mathrm{v}_{2} K^{(2)} b_{2}\right)}{\left(\mathrm{v}_{m} K^{m}+\mathrm{v}_{1} K^{(1)}+\mathrm{v}_{2} K^{(2)}\right)}\right)\right] \delta_{i j}
\end{array}\right.
$$

In the special case for $K^{(1)}=K^{(2)}=K^{m}=K, v^{m}=v$ these relations coincide with the solution (5).

The expressions obtained can be used to solve the problem of the competing influence of structural components on the properties of the matrix. In particular, for a three-phase matrix system, it is possible to determine in what ratio the concentrations of the phase components must be located, so that the average stresses in the matrix vanish. It follows from (6) that this will be satisfied under condition

$$
\frac{\mathrm{v}_{1}}{\mathrm{v}_{2}}=\frac{K^{(2)} b_{2}}{K^{(1)} b_{1}},
$$

that is, when the ratio of concentrations is inversely proportional to the ratio of free strains experienced by the phase components.

\section{CONCLUSION}

Using the self-consistent field method, the problem of competing effect of structural components on the matrix properties arising from phase transformations is solved. In addition, the method allows determining the average stresses in the elements of the microstructure through the elastic characteristics and concentrations of the matrix and

phase components, as well as free deformations of the phases undergoing volume transformations. The results of calculations in particular cases coincide with the results of other authors obtained earlier.

\section{REFERENCES}

[1] N.D. Nyashina, P.V. Trusov, "Modeling of martensitic transformations in steels: kinematics of the meso-level", PNRPU Mechanics Bulletin, No. 4, 118-151 (2014).

[2] I.V. Mishustin, A.A. Movchan, "Modeling of phase and structure transformations occurring in shape memory alloys under nonmonotonically varying stresses", Mechanics of Solids, vol. 49, No. 1, 27-39 (2014).

[3] J. D. Eshelby, Continual Theory of Dislocations (Publisher of Foreign Literature, Moscow, 1963).

[4] T.D. Shermergor, The Theory of Elasticity of Micro-inhomogeneous Media ( Nauka, Moscow, 1977).

[5] I.N. Bogachev, A.A. Vainshtein, S.D. Volkov, Statisticheskoe metallovedenie (Metallurgiia, Moscow, 1984). 